\title{
AN APPLICATION OF THE MAXIMUM PRINCIPLE TO THE GEOMETRY OF PLANE CURVES
}

\author{
HAROLD H. JOHNSON
}

\begin{abstract}
The maximum principle of control theory is used to find necessary and sufficient conditions for a plane curve, which has bounded piecewise continuous curvature and prescribed initial and terminal points and directions, to have minimal length. This result is used to prove that such a closed curve having length $L$ and curvature $k$ satisfying $|k| \leqq K$ can be contained in a circle of radius $R$, where $R \leqq L / 4-(\pi-2) / 2 K$.
\end{abstract}

The purpose of this note is to illustrate how a result in control theory, Pontryagin's maximum principle [1], can be used to study plane curves. In order to use this principle, we must assume that our curves have continuous first and piecewise continuous second derivatives. This will be assumed for all curves discussed in this paper. We shall say that such curves have "piecewise continuous curvature." The maximum principle is used to find necessary conditions for a plane curve, which has bounded piecewise continuous curvature and prescribed initial and terminal points and directions, to have minimal length. This result is applied to give the following inequality. If $C$ is a curve of length $L$ having piecewise continuous curvature $k(s)$ satisfying $|k| \leqq K$, then $C$ can be contained in a circle of radius $R$, where $R \leqq L / 4-(\pi-2) / 2 K$.

If $x(s)$ and $y(s)$ are the coordinates of any curve having curvature $k(s)$, then $x^{\prime}=\cos (u), y^{\prime}=\sin (u), u^{\prime}=k$, where $s$ is arc length and $u(s)$ is the angle which the unit tangent $\left(x^{\prime}, y^{\prime}\right)$ makes with the $x$-axis. Conversely, solutions of this system for given piecewise continuous $k(s)$ give curves having $s$ as arc length and $k$ as curvature.

TheOREM 1. Let $K>0,\left(x_{0}, y_{0}, u_{0}\right)$ and $\left(x_{1}, y_{1}, u_{1}\right)$ be given. Then among the curves $(x(s), y(s)), 0 \leqq s \leqq L$, having $x(0)=x_{0}, y(0)=y_{0}, u(0)=u_{0}$ and $x(L)=x_{1}, y(L)=y_{1}, u(L)=u_{1}$ and piecewise continuous curvature $k(s)$ satisfying $|k| \leqq K$, any one which has minimal length $L$ must consist of straight line segments and arcs of circles of radius $1 / K$.

Received by the editors March 22, 1973 and, in revised form, September 10, 1973. AMS (MOS) subject classifications (1970). Primary 53A05, 52A40, 49B10.

Key words and phrases. Plane curves, maximum principle, geometric inequalities. 
Proof. This may be regarded as a problem in control theory, namely, that of minimizing $L$ subject to the initial and terminal conditions, by use of a suitable control parameter $k$ satisfying $|k| \leqq K$. Pontryagin's maximum principle is applied as follows. Let $\psi_{1}, \psi_{2}, \psi_{3}$ be new auxiliary dependent variables. Form $H=\psi_{1} \cos (u)+\psi_{2} \sin (u)+\psi_{3} k$, and consider the equations $\psi_{1}^{\prime}=-\partial H / \partial x=0, \psi_{2}^{\prime}=-\partial H / \partial y=0, \psi_{3}^{\prime}=-\partial H / \partial u=\psi_{1} \sin (u)-\psi_{2} \cos (u)$.

Then, if the curve determined by $k(s)$ is optimal, there exists a nontrivial solution $\psi_{1}, \psi_{2}, \psi_{3}$ of these equations such that

$$
\begin{aligned}
& \max _{|k| \leqq K} H\left(x(s), y(s), u(s), k, \psi_{1}(s), \psi_{2}(s), \psi_{3}(s)\right) \\
& \quad=H\left(x(s), y(s), u(s), k(s), \psi_{1}(s), \psi_{2}(s), \psi_{3}(s)\right) .
\end{aligned}
$$

It follows that along any portion of the curve where $\psi_{3}(s) \neq 0, k=$ (sign $\left.\psi_{3}\right) K= \pm K$. Thus, $(x, y)$ is an arc of a circle of radius $1 / K$.

Suppose $\psi_{3}(s)=0$ for $s$ in some interval. Then $\psi_{3}^{\prime}=0=\psi_{1} \sin (u)-$ $\psi_{2} \cos (u)$, and since $\psi_{1}(s), \psi_{2}(s), \psi_{3}(s)$ cannot be trivial, either $\psi_{1} \neq 0$ or $\psi_{2} \neq 0$. But $\psi_{1}$ and $\psi_{2}$ satisfy $\psi_{1}^{\prime}=\psi_{2}^{\prime}=0$, so they are constants. It follows that $u$ is constant along such an arc, so $u^{\prime}=k=0$ there, hence such an arc is a straight line segment.

THEOREM 2. Let $C$ be a closed plane curve of length $L$ having piecewise continuous curvature $k(s)$ satisfying $|k| \leqq K$. Then $C$ may be contained in a circle of radius $R \leqq L / 4-(\pi-2) / 2 K$. This estimate is best possible.

Proof. Let $\Gamma$ be a circle of minimal radius $R$ containing $C$. Then $C$ and $\Gamma$ are tangent in at least two points.

Case 1. Suppose $C \cap \Gamma$ consists of two points $A$ and $B$. These must be ends of a diameter of $\Gamma$, or $\Gamma$ could be reduced still more. We may assume that $C$ has minimal length among all closed curves tangent to $\Gamma$ at the ends of a diameter, whose curvatures satisfy $|k| \leqq K$.

Then each portion of $C$, from $A$ to $B$ and then from $B$ back to $A$, must be a minimal solution to a problem of the type studied in Theorem 1 . By that theorem, $C$ consists of straight line segments and circular arcs. But then the convex hull of $C$ has a boundary of the same type and no longer length. Hence we may assume $C$ to be convex. Then its curvature never changes sign, and so all circular arcs are traversed in the same direction, relative to their centers.

Such a curve must pass through $A$ and $B$ along some circular arc, otherwise $C$ would cross the circle $\Gamma$. For the same reason, the curvature of this circular arc cannot be smaller than the curvature of $\Gamma$, so $R \geqq 1 / K$.

Let us consider, then, one arc of $C$ from $A$ to $B$. Its initial and terminal directions are perpendicular to the line $A B$, and it consists in a succession of circular arcs and line segments. 
The only changes in direction occur along the circular arcs, and these result in a change of $\pi$ radians. Let us translate these arcs in order to form one continuous semicircle $\Sigma$ starting at $A$ and going to a point $D$ on the line $A B$. Then we translate the line segments in any order to form a polygon $\Delta$ from $D$ to $B$. The distance from $D$ to $B$ is $2 R-2 / K$, so $\Delta$ must have length at least $2 R-2 / K$. Hence the total length of this part of $C$ from $A$ to $B$ is at least $\pi / K+2 R-2 / K$, and similarly for the other part of the curve back again from $B$ to $A$. Hence $L \geqq 2(\pi / K+2 R-2 / K)=4 R+$ $2(\pi-2) / K$, or $R \leqq L / 4-(\pi-2) / 2 K$. Notice that equality occurs when $C$ is shaped like a race-track, with two semicircles at $A$ and $B$ tangent to $\Gamma$, joined by straight line segments.

Case 2. Assume $\Gamma$ is tangent to $C$ in three points $A, B, D$, taken in clockwise order. (It may be tangent in more.) Let $O$ be the center of $\Gamma$, let $\lambda=\angle A O B, \mu=\angle B O D, \nu=\angle D O A$, where $\lambda+\mu+\nu=2 \pi$. Notice that these angles can be no larger than $\pi$, or else $\Gamma$ could be chosen smaller.

By the same reasoning as in Case 1 , we can assume $C$ has minimal length among all closed curves joining $A, B, D$ which are tangent to $\Gamma$ and satisfy the curvature inequality, and $C$ is convex. By Theorem 1, $C$ consists of arcs of circles of radii $1 / K$ and straight line segments.

Again we rearrange by translation the part of $C$ from $A$ to $B$, taking the circular arcs first and then the line segments. The circular arcs form an angular change of $\lambda$ and move $A$ to a point $A^{\prime}$ on the circle of radius $1 / K$ through $A$ tangent to $\Gamma$. Then by trigonometry,

$$
\begin{aligned}
\left|B A^{\prime}\right| & =(R-1 / K)\left(\sin ^{2}(\pi-\lambda)+(1+\cos (\pi-\lambda))^{2}\right)^{1 / 2} \\
& =\sqrt{ } 2(R-1 / K)(1-\cos (\lambda))^{1 / 2} .
\end{aligned}
$$

The same reasoning applied to $B D$ and $D A$ shows that $C$ must have length at least

$$
\begin{aligned}
L \geqq & \sqrt{ } 2(R-1 / K)\left[(1-\cos (\lambda))^{1 / 2}+(1-\cos (\mu))^{1 / 2}+(1-\cos (v))^{1 / 2}\right] \\
& +2 \pi / K .
\end{aligned}
$$

The critical point of the function on the right occurs when $\lambda=\mu=\nu=2 \pi / 3$ with the value $3 \sqrt{ } 3(R-1 / K)+2 \pi / K$. Since this is larger than its value $\sqrt{ } 2(R-1 / K) 2 \sqrt{ } 2+2 \pi / K=4(R-1 / K)+2 \pi / K$ at an endpoint such as $\lambda=0$, $\mu=\nu=\pi$ (Case 1 ), we find no improvement over the inequality obtained in Case 1.

Remarks. 1. We observed that $R \geqq 1 / K$, so combining this with the inequality of Theorem 2 we get $L \geqq 2 \pi / K$.

2. When $K \rightarrow \infty, R \leqq L / 4$, an inequality of J. C. Nitsche [2]. Theorem 2 is a generalization of Nitsche's inequality, in which the optimal path was a line segment traversed once in each direction. 
3. The technique used here would apply whenever an optimal problem has a rectilinear solution. For example, one could study $K$-triangles, defined as the closed curves passing through three given points with curvature bounded by $K$, and of minimal length.

4. The corresponding problem for higher dimensions seems to involve circular helixes, when both curvature and torsion are restricted.

\section{REFERENCES}

1. V. G. Boltjanskiĭ, Mathematical methods of optimal control, "Nauka", Moscow, 1966; English transl., Holt, Rinehart and Winston, New York, 1969. MR 34 \#7230.

2. J. C. Nitsche, The smallest sphere containing a rectifiable curve, Amer. Math. Monthly 78 (1971), 881-882. MR 45 \#480.

10900 Hunt Club Road, Reston, Virginia 22070

Department of Mathematics, University of Washington, Seattle, Washington 98195 